\title{
Chuvas intensas no Estado da Bahia ${ }^{1}$
}

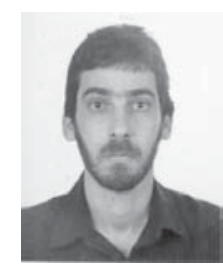

Demetrius D. da Silva², Raimundo R. Gomes Filho ${ }^{3}$, Fernando F. Pruski ${ }^{4}$, Sílvio B. Pereira ${ }^{5} \&$ Luciano F. de Novaes $^{6}$

\footnotetext{
1 Trabalho realizado mediante convênio firmado entre a Universidade Federal de Viçosa (UFV) e a Agência Nacional de Energia Elétrica (ANEEL)

2 DEA/UFV. Av. PH. Rolfs, Viçosa, MG. Fone: (31) 3899-1904. E-mail: david@ufv.br (Foto)

${ }^{3}$ DNOCS. Av. Coronel Solon, 330, Apt. 304, Fortaleza, CE. Fone: (85) 9602-6568. E-mail: rgomes@alunos.ufv.br ${ }^{4}$ DEA/UFV. Av. PH. Rolfs, Viçosa, MG. Fone: (31) 3899-1912. E-mail: ffpruski@ufv.br

5 DEA/UFV. Av. PH. Rolfs, Viçosa, MG. Fone: (31) 3899-1925. E-mail: sbueno@alunos.ufv.br

6 UFV. Av. PH. Rolfs, 305, Apt. 904, Viçosa, MG. Fone: (31) 3891-3572. E-mail: Ifnovaes@bol.com.br
}

Protocolo $064-8 / 5 / 2001$

\begin{abstract}
Resumo: Séries históricas de precipitação pluvial de 19 estações pluviográficas localizadas no Estado da Bahia e operadas pela Agência Nacional de Energia Elétrica (ANEEL), foram analisadas, objetivando-se ajustar modelos teóricos de distribuição de probabilidade aos dados de chuvas intensas e estabelecer a relação entre intensidade, duração e freqüência da precipitação pluvial. Para cada estação pluviográfica determinaram-se as séries de intensidade máxima anual das precipitações com durações de 10, 20, 30, 40, 50, 60, 120, 180, 240, 360, 720 e 1.440 min. Os modelos probabilísticos testados foram os de Gumbel, Log-Normal a dois e três parâmetros, Pearson e Log-Pearson III. As equações de intensidade-duração-freqüência da precipitação pluvial foram ajustadas utilizando-se o método de regressão não-linear de Gauss-Newton. $O$ teste de aderência de Kolmogorov-Smirnov, utilizado para a verificação do ajuste dos modelos aos dados de chuvas intensas, evidenciou que o modelo de Gumbel foi o que melhor se ajustou para a maior parte das combinações entre estações pluviográficas e durações estudadas. Foram evidenciadas, para uma mesma duração, grandes variações nas intensidades de precipitação entre as estações estudadas.
\end{abstract}

Palavras-chave: intensidade-duração-freqüência, estações pluviográficas, modelos probabilísticos

\section{High intensity rains in the Bahia State - Brazil}

\begin{abstract}
This study was conducted for fitting probabilistic models to data of rain storms. The intensity-duration-frequency relationships were established for 19 locations in Bahia State. Series with the annual maximum rainfall intensities for the durations of $10,20,30,40,50,60,120$, $180,240,360,720$ and $1440 \mathrm{~min}$ were used. Significant differences were observed in the maximum rainfall intensity values among the studied stations. The models utilized were Gumbel, Log-Normal (with two and three parameters), Pearson and Log-Pearson III. The KolmogorovSmirnov test was used to analyze the correlation between the model results and the rainfall data. The Gumbel model presented the best results for each duration. The intensity-duration-frequency equations were obtained using the Gauss-Newton method for non linear regression.
\end{abstract}

Key words: intensity-duration-frequency equation, precipitation stations, probabilistic models

\section{INTRODUÇÃO}

O conhecimento da equação que relaciona intensidade, duração e freqüência da precipitação pluvial, apresenta grande interesse de ordem técnica nos projetos de obras hidráulicas, como dimensionamento de vertedores, retificação de cursos d'água, galerias de águas pluviais, bueiros, sistemas de drenagem agrícola, urbana e rodoviária, dentre outros.
As dificuldades para a obtenção das equações de chuvas intensas decorrem de limitações referentes aos dados disponíveis, tanto em termos de densidade da rede pluviográfica, como em relação ao pequeno período de observações disponível; além disso, para a determinação dos parâmetros da equação de chuvas intensas é necessário exaustivo trabalho de análise, interpretação e codificação de grande quantidade de dados (Hernandez, 1991). 
A partir dos gráficos apresentados por Pfafstetter (1957), Denardin \& Freitas (1982) ajustaram equações matemáticas que possibilitaram o cálculo das alturas pluviométricas, em função da duração da precipitação e do período de retorno, utilizandose o método de regressão linear múltipla, para 80 estações pluviográficas distribuídas por todo o Brasil. Nesse estudo, no Estado da Bahia foi feita a determinação apenas para a cidade de Salvador. Froehlich (1993) descreveu um método gráfico considerando mapas de precipitações já disponíveis que permitiu obter-se, para qualquer localidade dos Estados Unidos, os parâmetros associados à várias formas de equações de intensidade de precipitação para durações de uma hora ou menos. Fendrich (1998) obteve as equações de chuvas intensas e gerou os gráficos de intensidade-duração-freqüência para 31 estações pluviográficas localizadas no Estado do Paraná. As séries históricas utilizadas no estudo possuíam períodos de 10 a 37 anos, com exceção da estação de Curitiba-Prado Velho (PUC), com apenas oito anos de dados. Pinto et al. (1996) obtiveram as equações de intensidade-duração-freqüência da precipitação pluvial para 29 estações pluviográficas do Estado de Minas Gerais, com base num período de 11 anos (19831993) com exceção de três estações, nas quais foi empregado um período-base de oito anos. Silva et al. (1999) estimaram os parâmetros da equação de intensidade-duração-freqüência da precipitação para 13 localidades do Estado do Rio de Janeiro e nove do Espírito Santo e realizaram, também, com base em técnicas de interpolação disponíveis em Sistemas de Informações Geográficas (SIG's), a espacialização dos parâmetros de ajuste da referida equação, para qualquer localidade dos dois Estados. Freitas et al. (2001) analisaram as séries históricas de precipitação pluvial de 193 estações pluviográficas localizadas no Estado de Minas Gerais e nos limites dos Estados da Bahia e Espírito Santo, objetivando ajustar modelos teóricos de distribuição de probabilidade aos dados de chuvas intensas e estabelecer a relação entre intensidade, duração e freqüência da precipitação pluvial, para essas estações. A análise dos resultados obtidos permitiu verificar-se que existe grande variabilidade das intensidades máximas ao longo do tempo, fato comprovado pelos altos desvios-padrão das séries anuais de intensidades máximas médias de precipitação pluvial encontrados para as diversas estações e durações estudadas. Verificaram, também, que o modelo de Gumbel foi o que apresentou melhor ajuste aos dados de intensidades máximas médias de precipitação pluvial pelo teste de Kolmogorov-Smirnov, a 20\% de probabilidade, para todas as durações estudadas.

Em decorrência da grande dificuldade na obtenção dos dados pluviográficos, a maioria dos estudos de chuvas intensas possui séries inferiores àquela recomendada pela Organização Mundial de Meteorologia (OMM), que é de 30 anos. Entretanto, Aron et al. (1987), objetivando determinar curvas regionais de intensidade, duração e freqüência de precipitação pluvial para o Estado da Pennsylvania (EUA) utilizaram séries históricas de 10 anos de duração. Já Button \& Ben-Asher (1983) utilizaram séries com oito anos de dados para obtenção da relação entre intensidade, duração e freqüência, na região de Avdat, Israel.

Segundo Aouad (1982) uma dificuldade imposta à análise da dinâmica do clima no Estado da Bahia é a sua própria posição geográfica, em face dos principais sistemas atmosféricos que ali atuam. $\mathrm{O}$ caráter transicional de seu espaço leva à dificuldade de identificar-se, com maior precisão, os sistemas meteorológicos que atingem essa área.

O território baiano é atingido por diferentes correntes de circulação, sendo que as de atuação mais efetivas se referem ao anticiclone semifixo do Atlântico Sul, notadamente pela sua periferia mais seca (alísios de sudeste) responsáveis, em parte, pela tendência à aridez no Estado da Bahia. Os alísios de sudeste atuam e ultrapassam o Estado da Bahia durante todo o ano, implicando em bom tempo, na maioria das vezes.

Um outro sistema mais complexo e que se configura também no corpo do anticiclone mencionado, refere-se às correntes perturbadas de leste, que produzem instabilidade e mau tempo. As correntes do sul constituem outra poderosa corrente de circulação; são geradas no anticiclone migratório polar, atravessando o Estado da Bahia, notadamente no setor litorâneo, precedidas pela Frente Polar Atlântica (FPA). A distância, consideravelmente grande, da sua fonte, faz com que essas correntes cheguem ao território baiano bastante tropicalizadas; entretanto, causam efeitos no regime pluviométrico da região. Do interior do continente pode atingir o território baiano outro tipo de corrente, também associada ao dinamismo de propagação da FPA.

$\mathrm{Na}$ faixa litorânea, a regularidade das precipitações é assegurada pela atuação de dois sistemas meteorológicos: as perturbações de leste, que produzem instabilidade e mau tempo, especialmente no setor setentrional, e as frentes frias, que causam chuvas frontais no setor litorâneo.

Devido a grande carência de informações relativas às equações de chuvas intensas para a maioria das localidades do Estado da Bahia, a alternativa para a realização de projetos de obras hidráulicas tem sido utilizar-se informações dos postos pluviográficos mais próximos da localidade na qual o projeto é realizado; este procedimento, entretanto, pode levar a estimativas pouco confiáveis, em função da grande variabilidade espacial dos dados de precipitação pluvial. Neste contexto, tendo em vista a importância que representa o conhecimento da equação que relaciona intensidade, duração e freqüência da precipitação pluvial para a realização de projetos hidroagrícolas, desenvolveu-se o presente trabalho, com os seguintes objetivos: (1) ajustar modelos teóricos de distribuição de probabilidade aos dados de chuvas intensas de 19 estações pluviográficas localizadas no Estado da Bahia, e (2) estabelecer a relação entre intensidade, duração e freqüência da precipitação, a partir dos registros pluviográficos das referidas estações.

\section{MATERIAL E MÉTODOS}

Neste trabalho foram utilizados os dados pluviográficos disponíveis para o Estado da Bahia, pertencentes à rede hidrometeorológica da Agência Nacional de Energia ElétricaANEEL, perfazendo 19 estações pluviográficas selecionadas (Tabela 1), com séries históricas de 10 a 24 anos de observações, abrangendo o período de 1975 a 1999. Ressalta-se que não foi adotado um período-base de estudos para todas as estações pois, ao se analisar os dados disponíveis para as estações 
Tabela 1. Caracterização das estações pluviográficas selecionadas para o Estado da Bahia

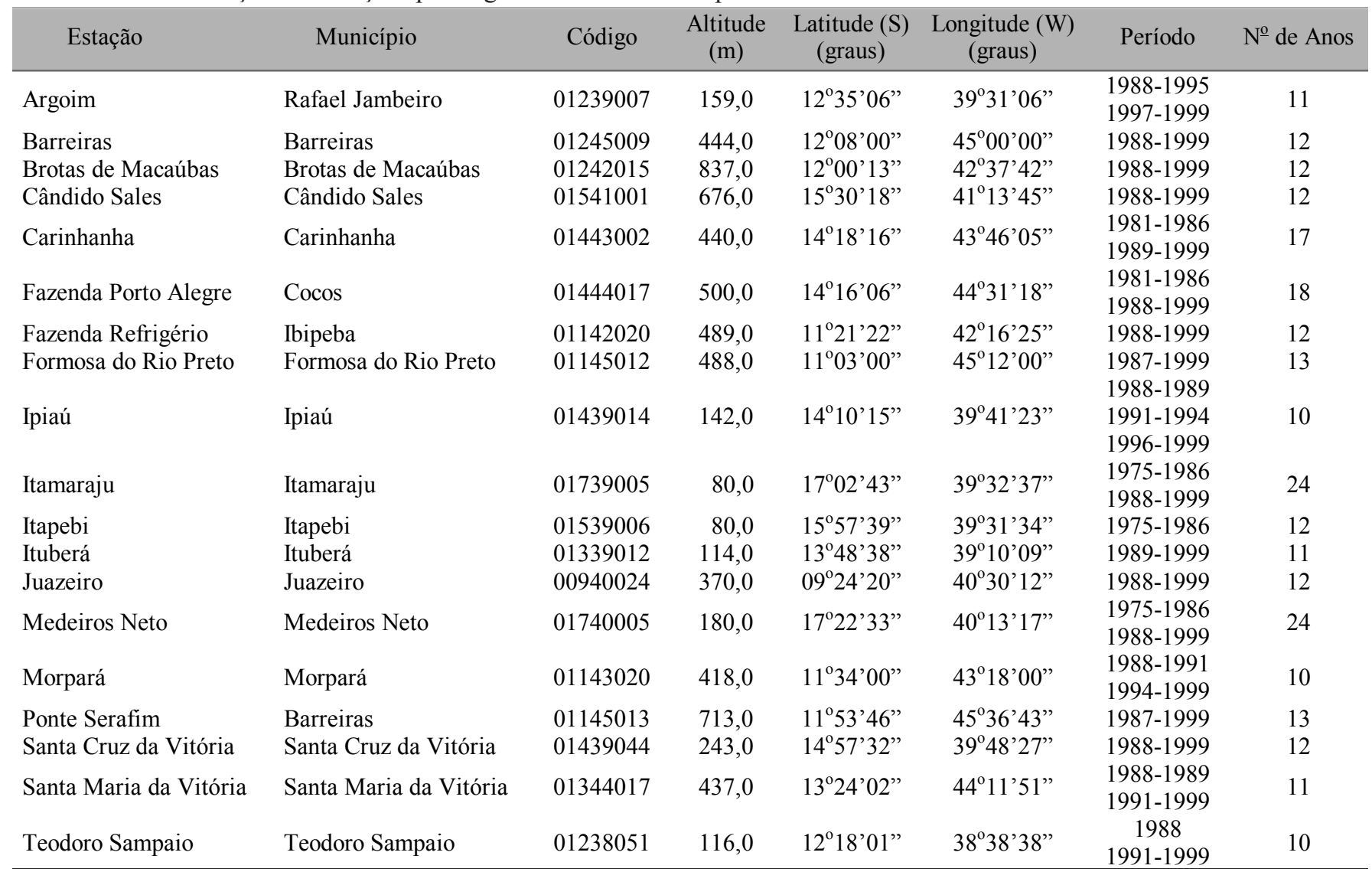

pluviográficas, verificou-se que elas não tinham períodos coincidentes. Assim, adotou-se como critério, o uso de todo o período de dados disponível para cada uma das estações visando-se, desta forma, aumentar o período de obtenção das informações sobre as chuvas intensas.

A equação utilizada para relacionar intensidade, duração e freqüência da precipitação pluvial apresentou a seguinte forma geral (Villela \& Mattos, 1975):

$$
i=\frac{k T^{a}}{(t+b)^{c}}
$$

em que:

i $\quad$ intensidade máxima média de chuva, $\mathrm{mm} \mathrm{h}^{-1}$

$\mathrm{T}$ - período de retorno, anos

t - duração da chuva, min

k, a, b, c - parâmetros empíricos que dependem da estação pluviográfica

O mapa do Estado da Bahia com a localização das estações pluviográficas utilizadas no trabalho é apresentado na Figura 1.

Os dados de precipitação pluvial foram obtidos com base nos pluviogramas que apresentaram chuvas mais intensas em cada ano do período estudado, cujo procedimento possibilitou a seleção de 879 pluviogramas. As séries históricas foram utilizadas sem o preenchimento de falhas, devido ao fato do uso de regressão linear, tanto simples como múltipla, ter apresentado baixos coeficientes de determinação. Foi realizada a leitura dos pluviogramas selecionados com o objetivo de se

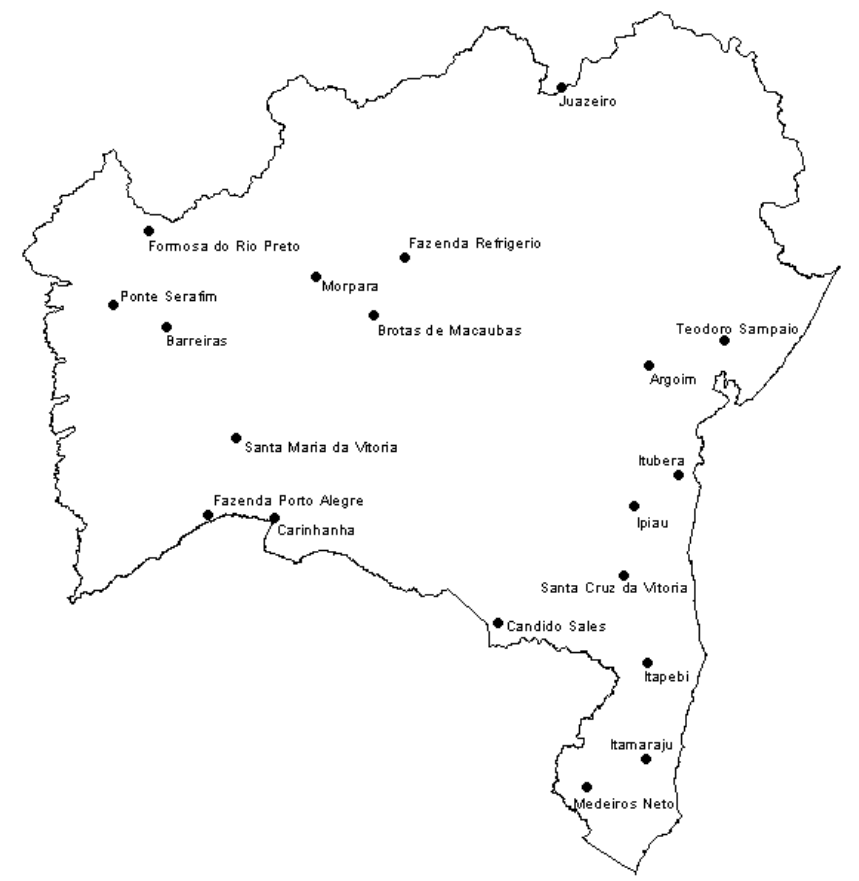

Figura 1. Mapa do Estado da Bahia com a localização das estações pluviográficas

obter as alturas pluviométricas máximas anuais nos tempos de $10,20,30,40,50,60,120,180,240,360,720$ e $1440 \mathrm{~min}$ em cada uma das estações selecionadas. Pela divisão das alturas pluviométricas máximas pela duração correspondente, obtevese a intensidade máxima média de precipitação. 
As séries históricas de intensidades máximas médias de precipitação pluvial, correspondentes às diversas durações, foram submetidas à análise estatística para identificação do modelo probabilístico que apresentasse melhor ajuste aos dados. Os modelos de distribuição de eventos extremos máximos ajustados, foram: Gumbel, Log-Normal a dois e três parâmetros, Pearson e Log-Pearson III e a seleção da distribuição de probabilidade que melhor se ajustou às séries históricas foi efetuada utilizando-se o teste de aderência de KolmogorovSmirnov. Utilizando-se o método de regressão não-linear Gauss-
Newton e com base nos valores de intensidade de precipitação máxima correspondentes aos períodos de retorno de 2, 5, 10, 20, 50 e 100 anos e duração de 10, 20,30, 40, 50, 60, 120, 240,360, 720 e 1.440 min, foram obtidos os parâmetros da equação de intensidade-duração-freqüência de cada estação pluviográfica.

\section{RESULTADOS E DISCUSSÃO}

Na Tabela 2 são apresentados as médias e os desvios-padrão das séries anuais de intensidades máximas de precipitação pluvial

Tabela 2. Médias ( $\overline{\mathrm{x}}$ e desvios-padrão $(\mathrm{s})^{*} \mathrm{em} \mathrm{mm} \mathrm{h}^{-1}$, das séries anuais de intensidades máximas médias de precipitação pluvial com duração de 10 a 1440 minutos, para as estações pluviográficas localizadas no Estado da Bahia

\begin{tabular}{|c|c|c|c|c|c|c|c|c|c|c|c|c|}
\hline \multirow{3}{*}{ Estação } & \multicolumn{12}{|c|}{ Duração (min) } \\
\hline & 10 & 20 & 30 & 40 & 50 & 60 & 120 & 180 & 240 & 360 & 720 & 1440 \\
\hline & $\overline{\mathrm{x}}$ & $\overline{\mathrm{x}}$ & $\overline{\mathrm{x}}$ & $\overline{\mathrm{x}}$ & $\overline{\mathrm{x}}$ & $\overline{\mathrm{x}}$ & $\overline{\mathrm{x}}$ & $\overline{\mathrm{x}}$ & $\overline{\mathrm{x}}$ & $\overline{\mathrm{x}}$ & $\overline{\mathrm{x}}$ & $\overline{\mathrm{x}}$ \\
\hline Argoim & $\begin{array}{c}95,1 \\
(34,9)\end{array}$ & $\begin{array}{c}75,1 \\
(30,8)\end{array}$ & $\begin{array}{c}61,9 \\
(28,0)\end{array}$ & $\begin{array}{c}53,3 \\
(27,3)\end{array}$ & $\begin{array}{c}45,4 \\
(24,7)\end{array}$ & $\begin{array}{c}39,7 \\
(22,8)\end{array}$ & $\begin{array}{c}22,6 \\
(14,4)\end{array}$ & $\begin{array}{c}16,3 \\
(10,6)\end{array}$ & $\begin{array}{l}12,8 \\
(8,2)\end{array}$ & $\begin{array}{c}9,0 \\
(5,5)\end{array}$ & $\begin{array}{c}4,8 \\
(3,0)\end{array}$ & $\begin{array}{c}2,5 \\
(1,5)\end{array}$ \\
\hline Barreiras & $\begin{array}{c}98,0 \\
(29,3)\end{array}$ & $\begin{array}{c}81,0 \\
(16,6)\end{array}$ & $\begin{array}{c}68,6 \\
(16,2)\end{array}$ & $\begin{array}{c}59,2 \\
(15,0)\end{array}$ & $\begin{array}{c}52,9 \\
(12,8)\end{array}$ & $\begin{array}{c}46,9 \\
(11,7)\end{array}$ & $\begin{array}{l}28,5 \\
(8,0)\end{array}$ & $\begin{array}{l}20,3 \\
(6,0)\end{array}$ & $\begin{array}{l}15,8 \\
(4,9)\end{array}$ & $\begin{array}{l}11,2 \\
(3,0)\end{array}$ & $\begin{array}{c}5,9 \\
(1,4)\end{array}$ & $\begin{array}{c}3,2 \\
(0,7)\end{array}$ \\
\hline Brotas de Macaúbas & $\begin{array}{c}91,7 \\
(26,3)\end{array}$ & $\begin{array}{c}73,9 \\
(19,0)\end{array}$ & $\begin{array}{c}60,9 \\
(17,5)\end{array}$ & $\begin{array}{c}52,2 \\
(16,1)\end{array}$ & $\begin{array}{c}45,1 \\
(13,9)\end{array}$ & $\begin{array}{c}38,7 \\
(12,1)\end{array}$ & $\begin{array}{l}21,0 \\
(7,0)\end{array}$ & $\begin{array}{l}15,1 \\
(5,4)\end{array}$ & $\begin{array}{l}11,7 \\
(4,3)\end{array}$ & $\begin{array}{c}8,4 \\
(3,2)\end{array}$ & $\begin{array}{c}4,4 \\
(1,7)\end{array}$ & $\begin{array}{c}2,2 \\
(0,9)\end{array}$ \\
\hline Cândido Sales & $\begin{array}{c}80,4 \\
(25,5)\end{array}$ & $\begin{array}{c}66,6 \\
(20,8)\end{array}$ & $\begin{array}{c}56,4 \\
(17,7)\end{array}$ & $\begin{array}{c}49,2 \\
(14,8)\end{array}$ & $\begin{array}{c}43,4 \\
(14,6)\end{array}$ & $\begin{array}{c}38,1 \\
(13,6)\end{array}$ & $\begin{array}{l}22,2 \\
(7,8)\end{array}$ & $\begin{array}{l}15,9 \\
(5,8)\end{array}$ & $\begin{array}{l}12,7 \\
(4,9)\end{array}$ & $\begin{array}{c}9,5 \\
(3,9)\end{array}$ & $\begin{array}{c}5,4 \\
(2,0)\end{array}$ & $\begin{array}{c}3,0 \\
(1,0)\end{array}$ \\
\hline Carinhanha & $\begin{array}{l}101,8 \\
(37,3)\end{array}$ & $\begin{array}{c}73,2 \\
(24,9)\end{array}$ & $\begin{array}{c}61,8 \\
(23,4)\end{array}$ & $\begin{array}{c}51,7 \\
(20,4)\end{array}$ & $\begin{array}{c}44,0 \\
(17,8)\end{array}$ & $\begin{array}{c}38,6 \\
(15,8)\end{array}$ & $\begin{array}{l}23,2 \\
(7,3)\end{array}$ & $\begin{array}{l}16,4 \\
(4,9)\end{array}$ & $\begin{array}{l}12,9 \\
(3,7)\end{array}$ & $\begin{array}{c}9,1 \\
(2,9)\end{array}$ & $\begin{array}{c}5,0 \\
(1,8)\end{array}$ & $\begin{array}{c}2,7 \\
(1,0)\end{array}$ \\
\hline Fazenda Porto Alegre & $\begin{array}{c}88,3 \\
(23,0)\end{array}$ & $\begin{array}{c}74,3 \\
(21,1)\end{array}$ & $\begin{array}{c}64,9 \\
(17,9)\end{array}$ & $\begin{array}{c}56,5 \\
(16,7)\end{array}$ & $\begin{array}{c}50,2 \\
(15,1)\end{array}$ & $\begin{array}{c}44,6 \\
(14,5)\end{array}$ & $\begin{array}{l}25,8 \\
(7,6)\end{array}$ & $\begin{array}{r}18,5 \\
(4,9)\end{array}$ & $\begin{array}{c}14,3 \\
(3,9)\end{array}$ & $\begin{array}{l}10,2 \\
(3,1)\end{array}$ & $\begin{array}{c}5,7 \\
(2,2)\end{array}$ & $\begin{array}{c}3,4 \\
(1,4)\end{array}$ \\
\hline Fazenda Refrigério & $\begin{array}{c}85,1 \\
(28,6)\end{array}$ & $\begin{array}{c}72,4 \\
(28,8)\end{array}$ & $\begin{array}{c}58,1 \\
(23,1)\end{array}$ & $\begin{array}{c}49,3 \\
(19,4)\end{array}$ & $\begin{array}{c}43,0 \\
(16,1)\end{array}$ & $\begin{array}{c}38,7 \\
(13,5)\end{array}$ & $\begin{array}{l}22,1 \\
(8,1)\end{array}$ & $\begin{array}{l}15,8 \\
(5,2)\end{array}$ & $\begin{array}{l}12,5 \\
(4,0)\end{array}$ & $\begin{array}{c}9,0 \\
(3,0)\end{array}$ & $\begin{array}{c}5,0 \\
(1,7)\end{array}$ & $\begin{array}{c}2,6 \\
(0,9)\end{array}$ \\
\hline Formosa do Rio Preto & $\begin{array}{c}96,4 \\
(25,8)\end{array}$ & $\begin{array}{c}74,5 \\
(16,1)\end{array}$ & $\begin{array}{c}64,7 \\
(16,2)\end{array}$ & $\begin{array}{c}55,7 \\
(13,4)\end{array}$ & $\begin{array}{c}47,9 \\
(12,1)\end{array}$ & $\begin{array}{c}41,4 \\
(10,4)\end{array}$ & $\begin{array}{l}24,5 \\
(6,3)\end{array}$ & $\begin{array}{l}18,0 \\
(5,2)\end{array}$ & $\begin{array}{l}14,2 \\
(4,1)\end{array}$ & $\begin{array}{l}10,0 \\
(2,7)\end{array}$ & $\begin{array}{c}5,6 \\
(1,6)\end{array}$ & $\begin{array}{c}3,0 \\
(0,9)\end{array}$ \\
\hline Ipiaú & $\begin{array}{c}87,5 \\
(30,6)\end{array}$ & $\begin{array}{c}70,8 \\
(28,1)\end{array}$ & $\begin{array}{c}57,6 \\
(24,3)\end{array}$ & $\begin{array}{c}51,4 \\
(20,9)\end{array}$ & $\begin{array}{c}44,1 \\
(18,5)\end{array}$ & $\begin{array}{c}39,5 \\
(16,4)\end{array}$ & $\begin{array}{c}25,0 \\
(11,5)\end{array}$ & $\begin{array}{c}18,8 \\
(10,0)\end{array}$ & $\begin{array}{r}14,7 \\
(7,6)\end{array}$ & $\begin{array}{l}10,4 \\
(4,9)\end{array}$ & $\begin{array}{c}5,3 \\
(2,5)\end{array}$ & $\begin{array}{c}2,8 \\
(1,3)\end{array}$ \\
\hline Itamaraju & $\begin{array}{c}89,6 \\
(32,8)\end{array}$ & $\begin{array}{c}69,2 \\
(27,5)\end{array}$ & $\begin{array}{c}57,1 \\
(23,8)\end{array}$ & $\begin{array}{c}48,5 \\
(18,2)\end{array}$ & $\begin{array}{c}42,2 \\
(14,6)\end{array}$ & $\begin{array}{c}37,4 \\
(12,5)\end{array}$ & $\begin{array}{l}23,2 \\
(7,0)\end{array}$ & $\begin{array}{l}16,7 \\
(4,8)\end{array}$ & $\begin{array}{l}13,2 \\
(3,7)\end{array}$ & $\begin{array}{c}9,5 \\
(2,6)\end{array}$ & $\begin{array}{c}5,3 \\
(1,5)\end{array}$ & $\begin{array}{c}3,2 \\
(0,9)\end{array}$ \\
\hline Itapebi & $\begin{array}{c}84,0 \\
(29,9)\end{array}$ & $\begin{array}{c}68,2 \\
(22,5)\end{array}$ & $\begin{array}{c}58,2 \\
(18,1)\end{array}$ & $\begin{array}{c}51,6 \\
(19,0)\end{array}$ & $\begin{array}{c}45,9 \\
(17,9)\end{array}$ & $\begin{array}{c}40,8 \\
(16,4)\end{array}$ & $\begin{array}{c}23,9 \\
(10,0)\end{array}$ & $\begin{array}{r}17,0 \\
(7,0)\end{array}$ & $\begin{array}{r}13,8 \\
(5,2)\end{array}$ & $\begin{array}{c}9,7 \\
(3,6)\end{array}$ & $\begin{array}{c}5,4 \\
(1,9)\end{array}$ & $\begin{array}{c}2,9 \\
(1,1)\end{array}$ \\
\hline Ituberá & $\begin{array}{c}79,8 \\
(25,9)\end{array}$ & $\begin{array}{c}65,3 \\
(17,7)\end{array}$ & $\begin{array}{c}54,7 \\
(15,2)\end{array}$ & $\begin{array}{c}50,1 \\
(17,5)\end{array}$ & $\begin{array}{c}45,0 \\
(17,8)\end{array}$ & $\begin{array}{c}40,0 \\
(16,9)\end{array}$ & $\begin{array}{c}24,5 \\
(8,8)\end{array}$ & $\begin{array}{l}18,6 \\
(6,5)\end{array}$ & $\begin{array}{l}14,9 \\
(5,1)\end{array}$ & $\begin{array}{l}11,1 \\
(3,8)\end{array}$ & $\begin{array}{c}6,5 \\
(1,7)\end{array}$ & $\begin{array}{c}3,9 \\
(1,1)\end{array}$ \\
\hline Juazeiro & $\begin{array}{c}80,0 \\
(35,7)\end{array}$ & $\begin{array}{c}65,0 \\
(29,5)\end{array}$ & $\begin{array}{c}57,5 \\
(23,9)\end{array}$ & $\begin{array}{c}49,2 \\
(21,1)\end{array}$ & $\begin{array}{c}41,9 \\
(18,8)\end{array}$ & $\begin{array}{c}36,9 \\
(17,5)\end{array}$ & $\begin{array}{l}21,7 \\
(9,3)\end{array}$ & $\begin{array}{l}16,0 \\
(7,2)\end{array}$ & $\begin{array}{l}12,7 \\
(5,4)\end{array}$ & $\begin{array}{c}8,8 \\
(3,6)\end{array}$ & $\begin{array}{c}4,6 \\
(1,9)\end{array}$ & $\begin{array}{c}2,4 \\
(1,0)\end{array}$ \\
\hline Medeiros Neto & $\begin{array}{c}95,3 \\
(39,6)\end{array}$ & $\begin{array}{c}78,3 \\
(33,0)\end{array}$ & $\begin{array}{c}66,1 \\
(28,0)\end{array}$ & $\begin{array}{c}55,8 \\
(25,1)\end{array}$ & $\begin{array}{c}48,2 \\
(22,0)\end{array}$ & $\begin{array}{c}42,5 \\
(19,2)\end{array}$ & $\begin{array}{c}24,9 \\
(10,8)\end{array}$ & $\begin{array}{c}18,4 \\
(8,2)\end{array}$ & $\begin{array}{l}14,4 \\
(6,2)\end{array}$ & $\begin{array}{l}10,2 \\
(4,2)\end{array}$ & $\begin{array}{c}5,5 \\
(2,1)\end{array}$ & $\begin{array}{c}3,0 \\
(1,1)\end{array}$ \\
\hline Morpará & $\begin{array}{c}79,8 \\
(32,4)\end{array}$ & $\begin{array}{c}69,8 \\
(26,8)\end{array}$ & $\begin{array}{c}54,9 \\
(21,4)\end{array}$ & $\begin{array}{c}48,2 \\
(18,2)\end{array}$ & $\begin{array}{c}42,2 \\
(15,8)\end{array}$ & $\begin{array}{c}37,3 \\
(14,4)\end{array}$ & $\begin{array}{l}22,1 \\
(9,7)\end{array}$ & $\begin{array}{r}16,4 \\
(8,9)\end{array}$ & $\begin{array}{r}13,7 \\
(7,8)\end{array}$ & $\begin{array}{c}9,7 \\
(5,5)\end{array}$ & $\begin{array}{c}5,0 \\
(2,7)\end{array}$ & $\begin{array}{c}2,5 \\
(1,4)\end{array}$ \\
\hline Ponte Serafim & $\begin{array}{c}84,6 \\
(23,9)\end{array}$ & $\begin{array}{c}72,0 \\
(19,9)\end{array}$ & $\begin{array}{c}58,8 \\
(14,4)\end{array}$ & $\begin{array}{c}47,5 \\
(11,7)\end{array}$ & $\begin{array}{c}40,5 \\
(10,3)\end{array}$ & $\begin{array}{l}35,5 \\
(8,8)\end{array}$ & $\begin{array}{c}19,8 \\
(5,0)\end{array}$ & $\begin{array}{l}14,4 \\
(4,1)\end{array}$ & $\begin{array}{l}11,2 \\
(3,4)\end{array}$ & $\begin{array}{c}7,6 \\
(2,4)\end{array}$ & $\begin{array}{c}4,3 \\
(1,3)\end{array}$ & $\begin{array}{c}2,5 \\
(0,8)\end{array}$ \\
\hline Santa Cruz da Vitória & $\begin{array}{c}85,3 \\
(33,0)\end{array}$ & $\begin{array}{c}72,0 \\
(32,2)\end{array}$ & $\begin{array}{c}61,4 \\
(26,1)\end{array}$ & $\begin{array}{c}52,4 \\
(23,2)\end{array}$ & $\begin{array}{c}44,5 \\
(20,3)\end{array}$ & $\begin{array}{c}39,0 \\
(17,9)\end{array}$ & $\begin{array}{l}21,6 \\
(9,6)\end{array}$ & $\begin{array}{l}15,5 \\
(6,1)\end{array}$ & $\begin{array}{l}12,2 \\
(4,4)\end{array}$ & $\begin{array}{c}9,1 \\
(3,2)\end{array}$ & $\begin{array}{c}4,9 \\
(1,6)\end{array}$ & $\begin{array}{c}2,6 \\
(0,9)\end{array}$ \\
\hline Santa Maria da Vitória & $\begin{array}{c}93,8 \\
(31,9)\end{array}$ & $\begin{array}{c}76,7 \\
(27,0)\end{array}$ & $\begin{array}{c}64,3 \\
(23,2)\end{array}$ & $\begin{array}{c}54,1 \\
(19,8)\end{array}$ & $\begin{array}{c}46,8 \\
(16,1)\end{array}$ & $\begin{array}{c}42,4 \\
(14,3)\end{array}$ & $\begin{array}{c}25,2 \\
(10,2)\end{array}$ & $\begin{array}{l}18,5 \\
(7,2)\end{array}$ & $\begin{array}{r}14,9 \\
(5,6)\end{array}$ & $\begin{array}{l}10,7 \\
(4,0)\end{array}$ & $\begin{array}{c}6,1 \\
(2,3)\end{array}$ & $\begin{array}{c}3,1 \\
(1,1)\end{array}$ \\
\hline Teodoro Sampaio & $\begin{array}{c}97,1 \\
(26,6)\end{array}$ & $\begin{array}{c}79,5 \\
(24,2)\end{array}$ & $\begin{array}{c}70,9 \\
(25,1)\end{array}$ & $\begin{array}{c}61,1 \\
(22,6)\end{array}$ & $\begin{array}{c}53,5 \\
(20,1)\end{array}$ & $\begin{array}{c}47,3 \\
(17,7)\end{array}$ & $\begin{array}{c}27,7 \\
(11,4)\end{array}$ & $\begin{array}{l}19,2 \\
(7,8)\end{array}$ & $\begin{array}{r}14,7 \\
(5,8)\end{array}$ & $\begin{array}{l}10,6 \\
(4,4)\end{array}$ & $\begin{array}{c}6,1 \\
(2,4)\end{array}$ & $\begin{array}{c}3,2 \\
(1,2)\end{array}$ \\
\hline Média & $\begin{array}{c}89,1 \\
(30,2)\end{array}$ & $\begin{array}{c}72,5 \\
(24,6)\end{array}$ & $\begin{array}{c}61,0 \\
(21,2)\end{array}$ & $\begin{array}{c}52,5 \\
(19,0)\end{array}$ & $\begin{array}{c}45,6 \\
(16,8)\end{array}$ & $\begin{array}{c}40,3 \\
(15,1)\end{array}$ & $\begin{array}{l}23,6 \\
(8,9)\end{array}$ & $\begin{array}{l}17,1 \\
(6,6)\end{array}$ & $\begin{array}{c}13,5 \\
(5,2)\end{array}$ & $\begin{array}{c}9,7 \\
(3,6)\end{array}$ & $\begin{array}{c}5,3 \\
(2,0)\end{array}$ & $\begin{array}{c}2,9 \\
(1,1)\end{array}$ \\
\hline Desvio & $\begin{array}{c}7,0 \\
(4,7)\end{array}$ & $\begin{array}{c}4,5 \\
(5,3)\end{array}$ & $\begin{array}{c}4,6 \\
(4,4)\end{array}$ & $\begin{array}{c}3,8 \\
(4,0)\end{array}$ & $\begin{array}{c}3,6 \\
(3,5)\end{array}$ & $\begin{array}{c}3,2 \\
(3,3)\end{array}$ & $\begin{array}{c}2,2 \\
(2,2)\end{array}$ & $\begin{array}{c}1,6 \\
(1,8)\end{array}$ & $\begin{array}{c}1,2 \\
(1,4)\end{array}$ & $\begin{array}{c}0,9 \\
(0,9)\end{array}$ & $\begin{array}{c}0,6 \\
(0,5)\end{array}$ & $\begin{array}{c}0,4 \\
(0,2)\end{array}$ \\
\hline $\mathrm{CV}(\%)^{* *}$ & $\begin{array}{c}7,8 \\
(15,6)\end{array}$ & $\begin{array}{c}6,3 \\
(21,6)\end{array}$ & $\begin{array}{c}7,5 \\
(20,6)\end{array}$ & $\begin{array}{c}7,2 \\
(20,9)\end{array}$ & $\begin{array}{c}7,9 \\
(21,1)\end{array}$ & $\begin{array}{c}8,0 \\
(22,1)\end{array}$ & $\begin{array}{c}9,4 \\
(24,5)\end{array}$ & $\begin{array}{c}9,3 \\
(27,4)\end{array}$ & $\begin{array}{c}9,2 \\
(27,3)\end{array}$ & $\begin{array}{c}9,7 \\
(25,1)\end{array}$ & $\begin{array}{c}11,3 \\
(23,5)\end{array}$ & $\begin{array}{c}14,3 \\
(20,8)\end{array}$ \\
\hline Relação max./min. ${ }^{* * *}$ & 1,28 & 1,25 & 1,30 & 1,29 & 1,32 & 1,33 & 1,44 & 1,41 & 1,42 & 1,47 & 1,52 & 1,76 \\
\hline
\end{tabular}

* Os valores entre parênteses referem-se ao desvio-padrão

** CV - Coeficiente de variação

*** A relação max/min expressa o quociente entre os valores externos de intensidade de precipitação máxima e mínima entre as localidades, para cada período 
correspondentes às durações estudadas, para cada uma das 19 estações do Estado da Bahia. Nesta tabela constam, também, para cada duração, as relações máxima/mínima entre os valores extremos de intensidade de precipitação máxima entre as diversas localidades.

Foram observados os menores valores de intensidade máxima média de precipitação pluvial para as durações de 10 e 30 min em Ituberá; Juazeiro, para 20 min; Brotas de Macaúba, para a duração de 1.440 min e Porto Serafim, para as demais durações. Por outro lado, maiores valores de intensidade máxima média da precipitação pluvial foram observados em Carinhanha, para a duração de 10 min; Barreiras, para as durações de 20, 120, 180, 240 e 360 min; Teodoro Sampaio, para as durações de 30, 40, 50 e 60 min e Ituberá, para 720 e 1.440 min. Observa-se, ainda, que Ituberá, estação localizada no litoral baiano, mesmo apresentando os menores valores de intensidade máxima média de precipitação pluvial para as pequenas durações (10 e $30 \mathrm{~min}$ ) foi também a localidade que apresentou as maiores intensidades de precipitação máxima média para as maiores durações (720 e $1.440 \mathrm{~min}$ ). Conforme relatado por Aouad (1982) as correntes de circulação que atuam no Estado da Bahia, oponentes em direção, conferem padrões diferenciados de comportamento atmosférico, gerando fragmentação complexa do seu território.

A análise das relações entre os valores extremos de intensidade máxima média de precipitação pluvial para cada duração revela valores crescentes com o aumento da duração. A relação entre os valores máximo e mínimo da intensidade máxima média de precipitação foi de 1,28, para a duração de 10 minutos, e de 1,76 para o tempo de $1.440 \mathrm{~min}$. Isso indica que os erros advindos da estimativa da intensidade máxima média para determinada localidade, considerando-se os dados pertinentes a outra localidade, tendem a crescer com o aumento da duração. Esse fato é também evidenciado a partir da análise dos coeficientes de variação obtidos para as diversas durações, os quais também apresentam tendência crescente com o aumento da duração da precipitação pluvial. Face às grandes diferenças observadas entre as intensidades máximas médias de precipitação obtidas nas diferentes localidades para cada duração, sobretudo as maiores, constata-se a necessidade de obtenção de equações que representem as condições das chuvas intensas para a localidade de interesse. Assim, a melhor maneira de minimizar as imprecisões na estimativa da intensidade máxima média de precipitação pluvial, é por intermédio da ampliação de estudos, como o proposto, para um número cada vez maior de localidades.

De acordo com o teste de Kolmogorov-Smirnov, os modelos teóricos de distribuição de probabilidade de Gumbel e Log-Normal a dois parâmetros, foram os que melhor se ajustaram, ao nível de significância de $20 \%$ de probabilidade, às séries de intensidades máximas anuais. $\mathrm{O}$ modelo de Gumbel apresentou melhor comportamento em número maior de ocorrências (considera-se "ocorrência" cada combinação de estação pluviográfica e duração da precipitação pluvial). O modelo Pearson tipo III apresentou, também, bom comportamento, mas em número menor de ocorrências. O modelo Log-Normal a três parâmetros apresentou ajuste adequado às séries de intensidades máximas, embora em número ainda mais reduzido que o modelo Pearson tipo III, enquanto o modelo Log-Pearson tipo III mostrou ajuste inadequado para a maioria das séries de intensidades máximas analisadas.
Na Tabela 3 são apresentadas as equações de intensidadeduração-freqüência para as localidades estudadas, com seus respectivos coeficientes de determinação. Observa-se que os

Tabela 3. Equações de intensidade máxima média de precipitação pluvial (i), em $\mathrm{mm} \mathrm{h}^{-1}$, em função do período de retorno (T), em anos, e da duração da precipitação (t), em minutos, para 19 estações pluviográficas do Estado da Bahia

\begin{tabular}{|c|c|c|}
\hline Estação & Equação & $\mathrm{R}^{2}$ \\
\hline Argoim & $\mathrm{i}=\frac{8999,000 \mathrm{~T}^{0,245}}{(\mathrm{t}+56,068)^{1,119}}$ & 0,990 \\
\hline Barreiras & $i=\frac{1525,758 \mathrm{~T}^{0,178}}{(\mathrm{t}+19,457)^{0,820}}$ & 0,992 \\
\hline Brotas de Macaúbas & $\mathrm{i}=\frac{4210,017 \mathrm{~T}^{0,192}}{(\mathrm{t}+32,453)^{1,042}}$ & 0,995 \\
\hline Cândido Sales & $\mathrm{i}=\frac{2828,391 \mathrm{~T}^{0,204}}{(\mathrm{t}+34,463)^{0,956}}$ & 0,995 \\
\hline Carinhanha & $i=\frac{2718,147 T^{0,214}}{(t+21,193)^{0,978}}$ & 0,996 \\
\hline Fazenda Porto Alegre & $\mathrm{i}=\frac{2500,000 \mathrm{~T}^{0,184}}{(\mathrm{t}+34,478)^{0,902}}$ & 0,996 \\
\hline Fazenda Refrigério & $\mathrm{i}=\frac{3950,000 \mathrm{~T}^{0,222}}{(\mathrm{t}+33,862)^{1,028}}$ & 0,993 \\
\hline Formosa do Rio Preto & $i=\frac{1719,054 \mathrm{~T}^{0,174}}{(\mathrm{t}+20,021)^{0,865}}$ & 0,994 \\
\hline Ipiaú & $i=\frac{2194,929 \mathrm{~T}^{0,232}}{(\mathrm{t}+32,891)^{0,882}}$ & 0,991 \\
\hline Itamaraju & $\mathrm{i}=\frac{4032,860 \mathrm{~T}^{0,211}}{(\mathrm{t}+28,605)^{1,060}}$ & 0,997 \\
\hline Itapebi & $i=\frac{3586,593 T^{0,204}}{(t+39,135)^{0,987}}$ & 0,996 \\
\hline Ituberá & $\mathrm{i}=\frac{3228,481 \mathrm{~T}^{0,207}}{(\mathrm{t}+45,386)^{0,948}}$ & 0,990 \\
\hline Juazeiro & $i=\frac{5592,554 \mathrm{~T}^{0,242}}{(\mathrm{t}+40,039)^{1,093}}$ & 0,992 \\
\hline Medeiros Neto & $i=\frac{6899,271 T^{0,227}}{(t+40,913)^{1,107}}$ & 0,997 \\
\hline Morpará & $i=\frac{1121,260 T^{0,233}}{(t+19,746)^{0,783}}$ & 0,990 \\
\hline Ponte Serafim & $i=\frac{4073,933 \mathrm{~T}^{0,181}}{(\mathrm{t}+27,902)^{1,073}}$ & 0,994 \\
\hline Santa Cruz da Vitória & $i=\frac{3450,000 T^{0,239}}{(t+34,012)^{0,989}}$ & 0,993 \\
\hline Santa Maria da Vitória & $\mathrm{i}=\frac{2873,405 \mathrm{~T}^{0,216}}{(\mathrm{t}+29,656)^{0,946}}$ & 0,994 \\
\hline Teodoro Sampaio & $\mathrm{i}=\frac{5850,000 \mathrm{~T}^{0,212}}{(\mathrm{t}+51,820)^{1,021}}$ & 0,994 \\
\hline
\end{tabular}


valores dos parâmetros de ajuste $(k, a, b, c)$ das equações variaram bastante de uma estação para outra. O valor do coeficiente k variou de 1121,260 a 8999,000, para as estações de Morpará e Argoim, respectivamente, o coeficiente a, de 0,174 a 0,245 , referentes às estações de Formosa do Rio Preto e Argoim, respectivamente, o coeficiente b variou de 19,457 a 56,068 , relativos às estações de Barreiras e Argoim, respectivamente e o coeficiente c variou de 0,783 a 1,119 , para as estações de Morpará e Argoim, respectivamente. Na maioria das estações, os maiores valores do coeficiente $b$ foram relacionados aos maiores valores do coeficiente $\mathrm{k}$. Ressalta-se que outras combinações de coeficientes podem ser obtidas para a relação entre intensidade, duração e freqüência, sem causar perda significativa na precisão dos resultados.

\section{CONCLUSÕES}

Os resultados aqui apresentados permitem as seguintes conclusões:

1. Existe grande variabilidade dos valores de intensidade máxima média de precipitação, para uma mesma duração, entre as localidades estudadas.

2. As relações obtidas dos valores extremos de intensidade máxima média de precipitação pluvial para cada duração apresentam comportamento crescente com o aumento da duração da precipitação pluvial.

3. Os erros advindos da estimativa da intensidade máxima média para determinada localidade, considerando-se os dados pertinentes a outra localidade, tendem a crescer com o aumento da duração da precipitação pluvial.

4. Os modelos teóricos de distribuição de probabilidade de Gumbel e Log-Normal a dois parâmetros, foram os que melhor se ajustaram às séries de intensidades máximas anuais, sendo o modelo de Gumbel o que apresentou melhor comportamento para a maior parte das combinações entre estações pluviográficas e durações estudadas.

\section{LITERATURA CITADA}

Aouad, M.S. Tentativa de classificação climática para o Estado da Bahia: uma análise quantitativa dos atributos locais, associada à análise qualitativa do processo genético. Rio de Janeiro: IBGE, 1982. 80p.
Aron, G.; Wall, D.J.; White, E.I.; Dunn, C.N. Regional rainfall intensity-duration-frequency curves for Pennsylvania. Water Resources Bulletin, Salt Lake City, v.23, n.2, p.479-485, 1987.

Button, B.J.; Ben-Asher, J. Intensity-duration relationships of desert precipitation at Audat-Israel. Journal of Arid Enviromentals, London, v.6, n.5, p.1-12, 1983.

Denardin, J.L.; Freitas, P.L. Características fundamentais da chuva no Brasil. Pesquisa Agropecuária Brasileira, Brasília, v.17, n.10, p.1409-1416, 1982.

Fendrich, R. Chuvas intensas para obras de drenagem no Estado do Paraná. Curitiba: Champangnat, 1998. 99p.

Freitas, A.J.; Silva, D.D.; Pruski, F.F.; Pinto, F.A.; Pereira, S.B.; Gomes Filho, R.R.; Teixeira, A.F.; Baena, L.G.N.; Mello, L.T.A.; Novaes, L.F. Equações de chuvas intensas no Estado de Minas Gerais. Belo Horizonte: Companhia de Saneamento de Minas Gerais: Universidade Federal de Viçosa, 2001. 65p.

Froehlich, D.C. Short-duration-rainfall intensity equations for drainage design. Journal of Irrigation and Drainage Engineering. New York, v.119, n.5, p.814-828. 1993.

Hernandez, V. Ainda as equações de chuvas intensas - pode-se generalizar? In: Simpósio Brasileiro de Recursos Hídricos, 9: Simpósio Luso Brasileiro de Hidráulica e Recursos Hídricos, 5, Rio de Janeiro, 1991. Anais... Fortaleza: Associação Brasileira de Hidrologia e Recursos Hídricos,1991. p.193-202.

Pfafstetter, O. Chuvas intensas no Brasil. Rio de Janeiro: Ministério da Viação e Obras Públicas; DNOS, 1957. 420p.

Pinto, F.A.; Ferreira, P.A.; Pruski, F.F.; Alves, A.R.; Cecon, P.R. Estimativa de chuvas intensas no Estado de Minas Gerais, utilizando-se registros diários. Revista de Engenharia Agrícola, v.16, n.2, p.8-21, 1996.

Silva, D.D.; Pinto, F.R.L.P.; Pruski, F.F.; Pinto, F.A. Estimativa e espacialização dos parâmetros da equação de intensidadeduração-freqüência da precipitação para os Estados do Rio de Janeiro e Espírito Santo. Revista de Engenharia Agrícola, v.18, n.3, p.11-21, 1999.

Villela, S.M.; Mattos, A. Hidrologia aplicada. São Paulo: Editora McGraw-Hill do Brasil Ltda., 1975. 245p. 